\title{
Cost-Consequence Analysis of Three Different Diagnostic Strategies in the First- and Second-Line Treatment of Locally Advanced or Metastatic Non-Small-Cell Lung Cancer
}

\author{
Giovanni Gancitano ${ }^{1}$, Roberto Ravasio ${ }^{2}$, Matteo Dionisi ${ }^{1}$, Diego Cortinovis ${ }^{3}$ \\ ${ }^{1}$ Medical \& Market Access Department, Roche Diagnostics SpA, Monza, Italy \\ ${ }^{2}$ Health Publishing \& Services Srl, Milan, Italy \\ ${ }^{3}$ Medical Oncology Unit, San Gerardo Hospital, Monza, Italy
}

\section{ABSTRACT}

BACKGROUND: Unlike the tissue one, liquid biopsy is a less invasive diagnostic method for the assessment of possible mutations of the tumor, based on the analysis of circulating free DNA (cfDNA) present in the plasma component of the blood. Because blood samples are easily obtainable, plasma biopsy is a non-invasive method, supplementing the more traditional biopsy techniques.

AIM: A cost-consequence analysis was conducted to compare the adoption of three different diagnostic strategies in the first- and second-line treatment of locally advanced or metastatic NSCLC: i) tissue strategy (only tissue biopsy for first and second line), ii) combined strategy (first line: tissue biopsy. If unknown, liquid biopsy; second line: liquid biopsy. If negative, tissue biopsy) and iii) potential strategy (first line: tissue biopsy. If unknown or tissue ineligible, liquid biopsy; second line: liquid biopsy. If negative, tissue biopsy).

METHODS: A decision-analytic model was developed considering the Italian NHS's perspective. We only evaluated direct medical costs (tissue biopsy, management of complications associated with tissue and liquid biopsies) borne by the NHS. The CCA was conducted over a time horizon of 1 year, assuming that for each patient with mNSCLC the diagnostic pathway (first- and second-line treatment) ended within such period. Key variables were tested in the sensitivity analysis. RESULTS: Considering both the first and the second line of treatment, the potential strategy constitutes the cost-effective alternative, characterized by an average cost per correctly identified case $(€ 685)$ lower than that estimated for the combined strategy $(€ 732)$ or for the tissue strategy $(€ 1,004)$. The potential strategy remains cost-effective, also considering the results referred to the first- or second-line treatment only.

CONCLUSION: The choice of a correct diagnostic strategy is crucial in order to optimize cancer therapies in the first- and second-line treatment of locally advanced or metastasized NSCLC. The addition to the diagnostic pathway of the liquid biopsy would correctly identify a greater number of cases, supporting the prescription of the best oncological therapy.
\end{abstract}

\section{Keywords}

Liquid biopsy, Tissue biopsy, Cost-consequence analysis, NSCLC, Italian NHS

\section{INTRODUCTION}

In Italy, lung carcinoma represents the third most frequently diagnosed tumors $(11 \%$ of the total of tumors), excluding skin cancers, and the first leading cause of death from cancer for males (27\%) and the third for females (11\%) [1]. In 2017, approximately 41,800 new cases were estimated, of which 28,000 related to males and 13,600 to females [1]. Since the early stages of lung cancer do not show any symptoms - or they show non-specific symptoms - at the time of diagnosis, the majority of patients show a locally advanced or metastatic disease [2].

Clinically, lung carcinoma can be distinguished into two types, which together describe over $95 \%$ of cancers affecting this organ: microcytoma - or Small-Cell Lung Cancer (SCLC) - which accounts for approximately $15 \%$ of lung carcinomas, and Non-Small-Cell Lung Cancer (NSCLC), which accounts for approximately the remaining 85\% [3]. The latter is once again differentiated into three main subtypes: adenocarcinoma, Squamous Cell Carcinoma

Corresponding author Giovanni Gancitano giovanni.gancitano@roche.com 
(SCC), and Large Cell Carcinoma (LCC). NSCLC can, therefore, be considered as a heterogeneous set of tumors, with different histological and biomolecular characteristics [4].

In the era of personalized treatments (targeted therapies), histocitopathological diagnosis is a priority in the process of selection of the most appropriate cancer treatment [5]. Therefore, this selection increasingly more often should be based on the correspondence between the drug profile and the specific mutation of the primary tumor [6,7]. For example, tests for EGFR (Epidermal Growth Factor Receptor) and ALK (Anaplastic Lymphoma Kinase) mutations are recommended to support oncologists in the choice of the first- and second-line treatment in non-squamous cell carcinomas [7]. This trend is expanding the screening of other biomolecular alterations, including ROS1, BRAF and MET, among others.

In the majority of cases, the histocitopathological diagnosis is made by examining a lung tissue sample taken by tissue biopsy. By means of particular techniques of preparation and staining of the sample, the pathologist can then make a diagnosis and identify the type of cancer [8]. The pathologist can also perform the subsequent molecular analyses on the material collected, in order to obtain more information about the prognosis and the predictability of effectiveness of a certain treatment. However, tissue biopsy poses several limitations, for example when it is not possible to acquire an adequate amount of tissue to conduct all the molecular analyses [9].

The targeted diagnostic/therapeutic approach to the tumor requires the integration of conventional histopathological diagnostics with molecular characterization of the tumor. It means that, in order to implement an individualized cancer therapy, the identification of the molecular alterations characterizing the tumor is necessary, in addition to the organic and morphological classification.

In the absence of a molecular analysis, the choice of treatment will be directed towards chemotherapy in combination with cisplatin, thus precluding - in case of a patient with a specific mutation - the benefits resulting from the administration of a targeted therapy [5]. Generally, the disease progression requires a second line of treatment, which is indicated only for patients with an acceptable Performance Status (PS) [5]. In addition, in the metastatic stage, lung cancer often develops resistance to the first-line therapy. For example, in patients with sensitizing EGFR mutations (classically, exon 19 deletion; point mutation in exon 21), the resistance to EGFR tyrosine kinase inhibitors can be determined by the onset of the EGFR T790M mutation [10], or by mechanisms of the by-pass track type, like the presence of amplification of the MET gene, or by a change in the carcinoma histology [9]. Therefore, every time the cancer recurs or progresses, it would be appropriate to conduct additional molecular analyses, in order to identify the most appropriate cancer treatment.

In patients with progressing NSCLC, the repetition of the tissue biopsy may result in an increased risk of complications related with the diagnostic surgical procedures, or it could not even be feasible, due to the clinical conditions (PS) of the patient. In the latter case, in the presence of a mutation, the patient may be precluded from the benefits of a targeted therapy. Therefore, a clinical unmet need emerges and it requires an alternative procedure for the tissue biopsy.

Unlike the tissue one, liquid biopsy is a less invasive diagnostic method for the assessment of possible mutations of the tumor, based on the analysis of circulating free DNA (cfDNA) present in the plasma component of the blood [11]. Because blood samples are easily obtainable, plasma biopsy is a non-invasive method, supplementing the more traditional biopsy techniques. Liquid biopsy, moreover, can trace the prevalent DNA-releasing tumor population, considering the presence of numerous cell clones present in the primary tumor and in the metastatic sites. It is, therefore, a more "dynamic" method, which can trace the evolutionary process of a tumor exposed to the various selective pressures determined by the selected systemic or local therapies. The COBAS ${ }^{\circledR}$ EGFR Mutation Test v2 (Roche Molecular Systems, Inc., henceforth $\mathrm{COBAS}^{\circledR}$ test) is an oncology test that can be performed on plasma samples (liquid biopsy), in addition to tissue ones, and which, if necessary, allows to avoid the risks related to tissue biopsy and to the poor availability of the sample, which are an obstacle to the adoption of the molecular test. The COBAS ${ }^{\circledR}$ test uses real-time PCR technology for the detection and identification of the mutations of the EGFR gene (identification of 42 mutations in exons 18, 19, 20 and 21) in the DNA derived from plasma or from tumor tissue in patients with NSCLC, and is designed as an aid in the selection of the patients suffering from this disease and eligible for the treatment with EGFR tyrosine kinase inhibitors.

When addressing the issue of sustainability and efficient allocation of health resources, serious attention should be paid not only to the clinical aspects but also to the economic impact possibly deriving from a chosen diagnostic pathway. 
The aim of the present cost-consequence analysis (CCA) was to evaluate the economic impact generated by the adoption of three different diagnostic strategies in the first- and second-line treatment of locally advanced or metastatic NSCLC (adenocarcinoma, squamous cell carcinoma and large cell carcinoma), henceforth mNSCLC: i) tissue strategy, ii) combined strategy and iii) potential strategy.

\section{METHODS}

\section{Analysis technique}

With a view to overall sustainability, it is therefore important to optimize the diagnostic strategy of a mNSCLC patient, identifying the most convenient one in terms of both clinical benefit and costs. In line with this objective, a cost-consequence analysis (CCA) [12,13] was conducted. In a CCA the economic impact is estimated of costs and effects, regardless of a formal assessment of cost effectiveness. In other words, a CCA is substantially a list of the significant costs and consequences of an intervention

\section{Study design}

The CCA was conducted from the Italian National Health System (NHS) perspective. A decision-analytic model was developed to compare the three different diagnostic strategies in the first- and second-line treatment of mNSCLC. The tissue and combined strategies describe the clinical practice, while the potential strategy represents a hypothetical diagnostic pathway.

The tissue strategy (Figure 1A) provides for the execution of the tissue biopsy only for patients eligible to perform the tissue diagnostic investigation and to receive the first line of treatment; no additional diagnostic investigation is provided for the rest of patients who are eligible for treatment, but not for tissue biopsy. In the event of disease progression (secondline treatment), the strategy provides for the execution of a second tissue biopsy for all patients eligible for the tissue re-biopsy and the second-line treatment [3]. For the other patients eligible for treatment, but not for the tissue re-biopsy, no diagnostic investigation is provided. The combined strategy (Figure 1B) provides for the execution of the tissue biopsy in all patients eligible for the diagnostic investigation and the first-line treatment; for all cases where it was not possible to determine the outcome from the first tissue biopsy, the liquid biopsy is also performed. For patients eligible for treatment, but not for a tissue biopsy, no further biopsy procedure is provided. Unlike the tissue strategy, in the event of disease progression (second-line treatment), the execution of the liquid biopsy in all patients eligible to receive the second-line treatment is provided. If the outcome of the liquid biopsy is negative, a successive tissue biopsy is performed on those patients eligible for tissue re-biopsy. The potential strategy (Figure 1C) is entirely similar to the combined one, with the exception of what happens to patients eligible for the first line of treatment, but not eligible to the tissue biopsy; these patients are subjected to liquid biopsy.

Only tissue and liquid biopsies costs and the cost of complications associated with tissue biopsy were considered. No cost of complications associated with liquid biopsy were assumed. Other direct medical costs (eg. cancer treatment) and indirect costs (eg. loss of productivity) were not evaluated. The CCA was conducted over a time horizon of 1 year, as-

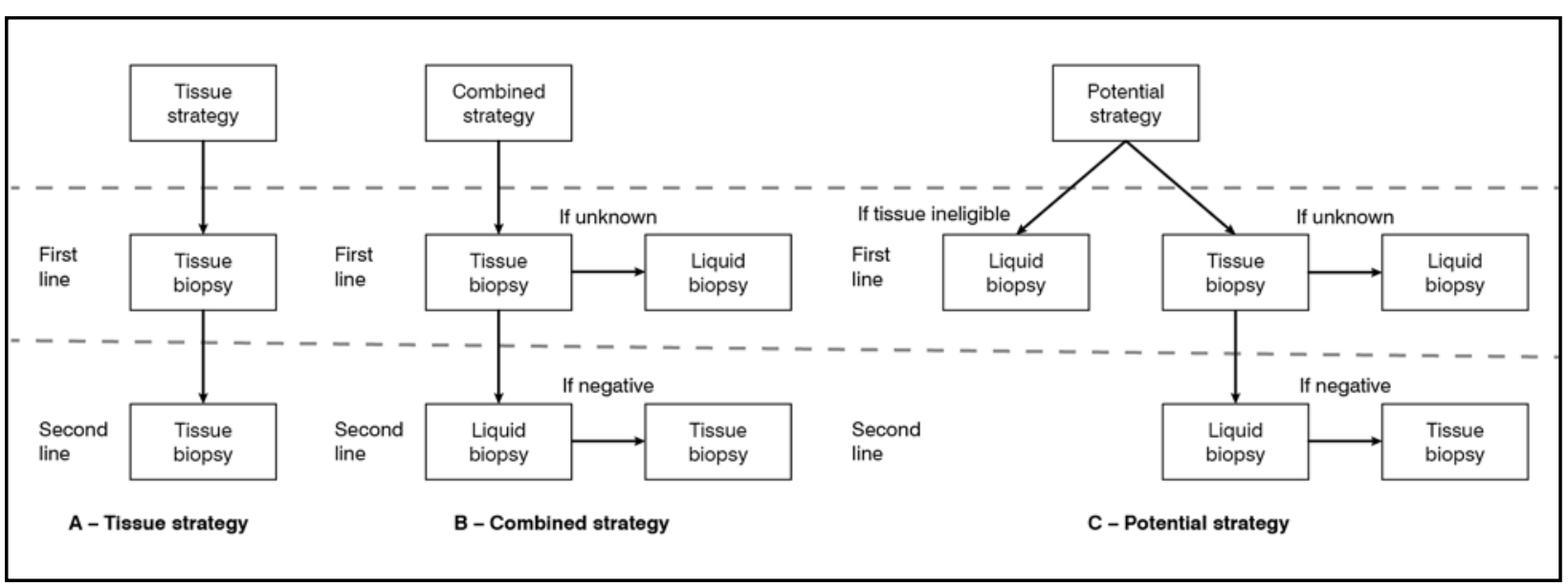

Figure 1. Diagnostic strategies compared in the analysis 


\begin{tabular}{lcc}
\hline \multicolumn{1}{c}{ Parameters } & Population (n.) & Source \\
\hline New patients with lung cancer (year 2017) & 41,800 & {$[1]$} \\
Patients with NSCLC (83.0\%) & 34,694 & {$[3]$} \\
Patients with adenocarcinoma, squamous cell carcinoma or large cell carcinoma (67.0\%) & 23,245 & {$[14]$} \\
Patients with locally advanced or metastasized diagnosis (74.1\%) & 17,225 & {$[15]$} \\
First-line treatment & & {$[3]$} \\
Patients eligible for the first-line treatment (89.0\%) & 15,330 & [2] \\
Patients eligible for the tissue biopsy (85.0\%) & 13,030 & Calculated from [16] \\
- Determinable outcome (70.0\%) & 9,121 & Calculated from [2] \\
- Non-determinable outcome (30.0\%) & 3,909 & [3] \\
Patients not eligible for tissue biopsy, but eligible for liquid biopsy (15.0\%) & 2,299 & [5] \\
Second-line treatment & 6,890 & Assumption \\
Patients eligible for the second-line treatment (40.0\%) & 5,650 & 6,890 \\
Patients eligible for the tissue re-biopsy (82.0\%) & & \\
Patients eligible for the liquid biopsy (100.0\%) & & \\
\hline
\end{tabular}

Table I. Estimated number of patients included in the CCA

\begin{tabular}{lcc|cc}
\hline \multirow{2}{*}{$\begin{array}{c}\text { EGFR } \\
\text { mutations }\end{array}$} & \multicolumn{2}{c|}{ Liquid biopsy } & \multicolumn{2}{c}{ Tissue biopsy } \\
\cline { 2 - 5 } & $\begin{array}{c}\text { Sensitivity } \\
\text { (\%) }\end{array}$ & $\begin{array}{c}\text { Specificity } \\
\text { (\%) }\end{array}$ & $\begin{array}{c}\text { Sensitivity } \\
\text { (\%) }\end{array}$ & $\begin{array}{c}\text { Specificity } \\
\text { (\%) }\end{array}$ \\
\hline Exons 19, 21 & 95.0 & 91.0 & 98.1 & 99.3 \\
T790M & 93.0 & 92.0 & 90.0 & 98.0 \\
\hline
\end{tabular}

Table II. Percentages sensitivity and specificity associated with the COBAS ${ }^{\circledR}$ EGFR Mutation Test [17,18] suming that for each patient with mNSCLC the diagnostic pathway (first- and second-line treatment) ended within such period. This hypothesis is certainly a limitation with respect to what happens in clinical practice - where patients with different prognoses are characterized by different treatment durations - but it allows to estimate the potential financial impact of the three strategies against a specific time period, which is a crucial element in the decision-making processes.

\section{Input data of the model}

\section{Population}

The estimated number of patients included in the CCA, reported in detail in Table I, was calculated starting from the number of new cases of lung cancer expected for 2017 in Italy (sample size) [1]. From these, the cases of NSCLC were first calculated [3], and then those with a diagnosis of adenocarcinoma, squamous cell carcinoma or large cell carcinoma were identified [14]. Then, only the cases relating to subjects with locally advanced or metastasized diagnosis [15] eligible for the first line of treatment [3] were considered. It is estimated that $85 \%$ of these patients are eligible for the tissue biopsy [2], while the remaining $15 \%$ potentially to the liquid biopsy only. Among the patients who perform the tissue biopsy, the outcome may not be determinable in $30 \%$ of cases [16]. Finally, $40 \%$ of patients with a mNSCLC diagnosis are eligible to receive the second-line treatment [3]; of them, $82 \%$ are eligible for the tissue re-biopsy [5] and $100 \%$ for the liquid one.

\section{Clinical data}

Table II shows the percentages of sensitivity and specificity associated with the COBAS ${ }^{\circledR}$ test performed on tissue (tissue biopsy) or plasma (liquid biopsy) samples and considered here $[17,18]$. In addition to these data, in the model - based on what is indicated in the literature - in the presence of a tissue biopsy, a $14 \%$ probability that the diagnostic investigation could determine the onset of a post-procedure complication (eg. pneumothorax) was considered [19]. The likelihood of such occurrence was set equal to $0 \%$ in the presence of a liquid biopsy.

\section{Biopsy cost and complications}

Table III shows the costs associated with the implementation of the tissue or liquid biopsy. The tissue biopsy provides for the execution of the biopsy of the pleura or the bronchus and the subsequent procedures aimed at determining the presence of DNA mutations (DNA mutation analysis, DNA digestion with restriction enzymes and extraction of DNA or RNA). In the case of the liquid biopsy, instead, in addition to the execution of the activities aimed at 
determining the presence of DNA mutations, only the sampling of venous blood is provided for. Every single specialist outpatient service was then attributed a value, considering the respective fee refunded by the NHS [20]. Thus, it was then estimated an average cost of $€ 412.02$ for the tissue biopsy and of $€ 233.86$ for the liquid one.

The onset of a post-tissue biopsy complication was instead esteemed considering a value of $€ 2,541.50$, obtained as a simple average between the amounts reimbursed for DRG 094 (pneumothorax with complications: $€ 3,265$ ) and DRG 095 (pneumothorax

\begin{tabular}{lcc}
\hline \multirow{2}{*}{\multicolumn{1}{c}{ Procedures }} & \multicolumn{2}{c}{ Unit cost (€) } \\
\cline { 2 - 3 } & $\begin{array}{c}\text { Tissue } \\
\text { biopsy }\end{array}$ & $\begin{array}{c}\text { Liquid } \\
\text { biopsy }\end{array}$ \\
\hline 34.24 Pleural or 33.24 bronchial biopsy & 180.74 & 0.00 \\
91.49.2 Venous blood sampling & 0.00 & 2.58 \\
DNA mutations & 231.28 & 231.28 \\
• 91.29.4 DNA mutation analysis & 120.80 & 120.80 \\
• 91.36.4 DNA digestion with restriction enzyme & 51.43 & 51.43 \\
• 91.36.5 Extraction of DNA or RNA & 59.05 & 59.05 \\
Total & $\mathbf{4 1 2 . 0 2}$ & $\mathbf{2 3 3 . 8 6}$ \\
\hline
\end{tabular}

Table III. Costs associated with the tissue and liquid biopsy without complications: $€ 1,818)[21]$.

\section{Output data of the model}

\section{Cost-effectiveness ratio (CER)}

On the basis of the eligible population and the estimated average cost for the (tissue and/ or liquid) biopsy and for the complications, the CCA provides - for the three diagnostic strategies, over a time horizon of one year - the number of eligible patients, the number of correctly identified cases and the total cost of the strategies. The difference in the spending borne by the NHS generated by the three different diagnostic strategies is presented in terms of average cost per correctly identified case (CER).

\section{Sensitivity analysis}

The sensitivity analysis was conducted with the aim of evaluating the degree of uncertainty of the base case results [22]. A first part of the sensitivity analysis was carried out by varying some epidemiological data used - in the base case - to estimate the number of the cases eligible for the three different diagnostic strategies. Specifically, with reference to the first line of treatment, and differently from what was done in the base case it has been hypothesized that all patients are eligible for the tissue biopsy (from $85 \%$ of the base case to $100 \%$ ). It was also verified the impact associated with the reduction in the percentage of cases where the outcome cannot be determined after the first tissue biopsy, actually halving the probability adopted in the base case (from $30 \%$ to $15 \%$ ). With reference to the second-line treatment, it was finally assumed a scenario in which the percentage of the cases eligible for the liquid biopsy was reduced, and set equal to that used for the tissue re-biopsy (82\%).

The second part of the sensitivity analysis was focused on the clinical data. In particular, compared to the base case, the sensitivity and specificity of the liquid biopsy in determining EGFR mutations (T790M and exons 19 and 21) was varied, by simultaneously considering (multivariate analysis) the respective lower limits of the confidence interval [17], while leaving unchanged the values of sensitivity and specificity associated with the tissue biopsy. With regard to the presence of the post-tissue biopsy complications, conservatively with respect to the base case, a 50\% reduction in complications (from 14\% to 7\%) was assumed.

The third and final part of the sensitivity analysis was conducted on the cost used to assign a value to the management of post-tissue biopsy complications. Conservatively, the lowest value $(€ 1,818)$ associated with the DRG 095 expected in case of pneumothorax without complications was considered.

\section{RESULTS}

\section{Economic impact}

Considering both the first and the second line of treatment, the potential strategy constitutes the cost-effective alternative, characterized by an average cost per correctly identified case (€ 685) lower than that estimated for the combined strategy ( $€ 732)$ or for the tissue strategy $(€ 1,004)$ (Table IV). This is due to the greater use of the liquid biopsy, which allows the potential strategy to significantly increase the number of correctly identified cases $(+10.9 \% \mathrm{vs}$ combined strategy; $+50.5 \%$ vs. tissue strategy), against a slight increase in the spending borne by the NHS $(+3.8 \%$ vs combined strategy; $+2.7 \%$ vs tissue strategy) (Table IV and Figure 2$)$. The potential strategy remains cost-effective, also considering the results referred to the firstor second-line treatment only. 


\begin{tabular}{|c|c|c|c|}
\hline & $\begin{array}{l}\text { Tissue } \\
\text { strategy }\end{array}$ & $\begin{array}{l}\text { Combined } \\
\text { strategy }\end{array}$ & $\begin{array}{l}\text { Potential } \\
\text { strategy }\end{array}$ \\
\hline \multicolumn{4}{|c|}{ First- and second-line treatment } \\
\hline Eligible patients (n.) & 18,680 & 19,920 & 22,220 \\
\hline Correctly identified cases (n.) & 14,288 & 19,394 & 21,504 \\
\hline Total cost $(€)$ & $14,343,085$ & $14,190,174$ & $14,727,930$ \\
\hline $\begin{array}{l}\text { Average cost per correctly } \\
\text { identified case }(€)\end{array}$ & 1,004 & 732 & 685 \\
\hline \multicolumn{4}{|l|}{ First-line treatment } \\
\hline Eligible patients (n.) & 13,030 & 13,030 & 15,330 \\
\hline Correctly identified cases (n.) & 9,037 & 12,624 & 14,733 \\
\hline Total cost $(€)$ & $10,005,112$ & $10,919,297$ & $11,457,052$ \\
\hline - Tissue biopsy cost $(€)$ & $5,368,774$ & $5,368,774$ & $5,368,774$ \\
\hline - Liquid biopsy cost (€) & 0 & 914,185 & $1,451,941$ \\
\hline $\begin{array}{l}\text { - Post-tissue biopsy } \\
\text { complications cost }(€)\end{array}$ & $4,636,337$ & $4,636,337$ & 4,636,337 \\
\hline $\begin{array}{l}\text { Average cost per correctly } \\
\text { identified case }(€)\end{array}$ & 1,107 & 865 & 778 \\
\hline \multicolumn{4}{|l|}{ Second-line treatment } \\
\hline Eligible patients (n.) & 5,650 & 6,890 & 6,890 \\
\hline Correctly identified cases (n.) & 5,251 & 6,770 & 6,770 \\
\hline Total cost $(€)$ & $4,337,973$ & $3,270,878$ & $3,270,878$ \\
\hline Tissue biopsy cost (€) & $2,327,770$ & 890,560 & 890,560 \\
\hline - Liquid biopsy cost (€) & 0 & $1,611,253$ & $1,611,253$ \\
\hline $\begin{array}{l}\text { - Post-tissue biopsy } \\
\text { complications cost }(€)\end{array}$ & $2,010,203$ & 769,065 & 769,065 \\
\hline $\begin{array}{l}\text { - Average cost per correctly } \\
\text { identified case }(€)\end{array}$ & 826 & 483 & 483 \\
\hline
\end{tabular}

Table IV. Results
With regard to the first line of treatment (Table IV), the patients eligible for the potential strategy are 15,330, while 13,030 are those eligible for the tissue or combined strategy. The difference of 2,299 patients $(17.6 \%)$ is due to the extension of use of the liquid biopsy for the subjects eligible for the cancer therapy, but not eligible for the tissue biopsy. By virtue of this, the number of correctly identified cases - with respect both to the combined strategy $(14,733$ vs. 12,624$)$ and the tissue strategy $(14,733$ vs. 9,037$)-$ increases. Given this greater effectiveness, despite an increase in costs $(+4.9 \%$ vs combined strategy; $14.5 \%$ vs tissue strategy), the potential strategy - with an average cost per correctly identified case of $€ 778$ - determines the most efficient allocation for the NHS.

Even considering the second-line treatment only (Table IV), the number of eligible patients differs between the three diagnostic strategies: 5,650 for the tissue strategy and 6,890 for the combined or potential ones. The difference (1,240 patients) is due to the different approach adopted by the strategies: in the tissue strategy, only the use of the tissue biopsy is provided for, while in the other two strategies the use of the liquid biopsy is called for, followed - if unsuccessful - by the execution of a subsequent tissue biopsy. The approach followed by the combined or potential strategy allows determining a greater number of correctly identified cases, compared to the tissue strategy $(6,770$ vs 5,$251 ;+28.9 \%)$. Unlike the estimates for the first line of treatment, the tissue strategy determines the higher costs borne by the NHS (€ 4,337,973 vs $€$ $3,270,878 ;+32.6 \%)$. Therefore, against a greater effectiveness and lower costs, in the second line of treatment, the combined and potential strategies are dominant compared to the tissue one (Table IV).

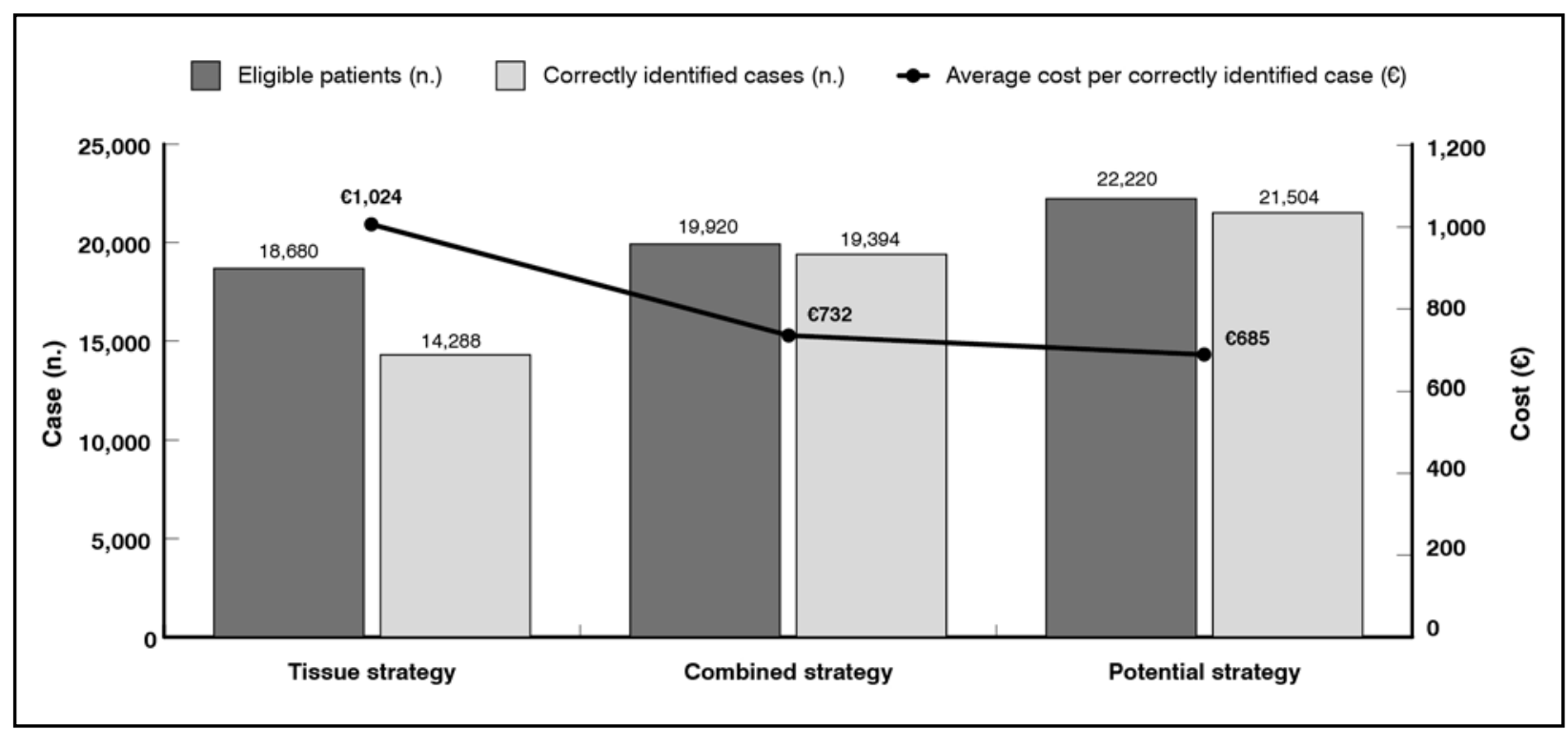

Figure 2. Results for first- and second-line treatment 


\section{Sensitivity analysis}

Table V shows the results of the sensitivity analysis, expressed in terms of average total cost (first and second line of treatment) per correctly identified case. In all comparisons, the potential strategy remains cost-effective.

Some results produced by the sensitivity analysis, however, deserve some thought. Both the reduction in the percentage of patients with post-tissue biopsy complications and the decrease in the average cost considered to assign a value to such eventuality affect significantly the base case results, reducing both the average cost per correctly identified case and the relative differences (Table V). The reduction of the probability that a patient in the first line can have a tissue biopsy with undetermined outcome affects predominantly the results of the comparison between tissue and combined strategy, reducing by more than $30 \%$ the difference between the two average costs compared to the base case (Table V). Finally, the adoption of the hypothesis that in the first line of treatment all patients are eligible for the tissue biopsy affects mainly the potential strategy, making it - in actual fact - equivalent to the combined one (the subjects undergoing the liquid biopsy following an undetermined outcome of the previous tissue biopsy would in fact be excluded).

\section{DISCUSSION}

A CCA was conducted, in order to assess the financial impact on the Italian NHS resulting from the adoption of three different diagnostic strategies in the first- and second-line treatment of mNSCLC. The decision-analytic model allowed to estimate, for each of the three alternative strategies, the clinical and economic consequences in terms of average cost per correctly identified case.

The potential strategy reported a lowest average cost per correctly identified case (€ 685) compared to the combined or tissue strategies ( $€ 732$ and $€ 1,004$, respectively). This result is due to the greater number of patients that the potential strategy allows to correctly identify compared to the two alternatives $(+10.9 \%$ vs combined strategy; $+50.5 \%$ vs. tissue strategy), against a slight increase in expenditure $(+3.8 \%$ vs combined strategy; $+2.7 \%$ vs tissue strategy). By limiting the observation to the comparison between the tissue and the combined strategy, it is evident that the latter is dominant, with a larger number of correctly diagnosed cases $(5,106)$ and a lower spending $(-152,910 €)$.

As it happens whenever the use of a simulation model is necessary, it is appropriate to interpret the results in light of a few considerations. The epidemiological analysis, on which this CCA is based, was conducted with the objective of defining, from a common sampling frame (number of new cases of lung cancer), the number of patients eligible for the three diagnostic strategies investigated. This process was conducted using data from different bibliographic sources and, in the absence of them, making some assumptions. To limit the uncertainty associated with this process, a wide sensitivity analysis was conducted on the major epidemiological variables that can affect the outcome of the base case. If in the first-line treatment the percentage of patients eligible for tissue biopsy switched from $85 \%$ (base case) to $100 \%$, the combined and potential strategies would lead to the same average cost per correctly identified case, while remaining both cost-effective compared to the tissue strategy. While it would not change the results of the base case, the reduction (from $30 \%$ to 15\%), in the first-line treatment, of the probability that a patient may have a tissue biopsy with an undetermined outcome would result in a significant decrease in the average cost per correctly identified case associated with each of the three strategies. Whereas the reduction in the second-line treatment of the percentage of patients eligible for the liquid biopsy (from $100 \%$ to $82 \%$ ) would not alter at all the results of the base case.

The sensitivity analysis - conducted on the sensitivity and specificity of the COBAS ${ }^{\circledR}$ test and on the probability that a complication related to the tissue biopsy could occur - confirmed 
in the first case the results of the base case, while in the second case it showed a significant reduction in the three average costs of per correctly identified case, confirming, however, the cost-effectiveness of the potential strategy.

The adoption of a more conservative criterion to assign a value to the management of the post-tissue biopsy complication (DRG 095 pneumothorax without complications: $€ 1,818$ ) would confirm, for the potential strategy, the lower average cost per correctly identified case.

Finally, it is to be emphasized that this analysis assessed only the consequences arising from the choice of one of the three different diagnostic strategies, measured in terms of costs per biopsy/complication and per correctly identified case, net of the consequences that this choice could have on the cost of the cancer treatment or on survival (overall or progression-free) of the patient. The possibility of carrying out a molecular analysis each time it is necessary, regardless of the patient's Performance Status, would allow to identify the most appropriate cancer treatment, thus consenting to maximize the effectiveness of the treatment pathway and minimize the waste of healthcare resources.

\section{CONCLUSIONS}

The results found here show how the choice of a correct diagnostic strategy is crucial in order to optimize cancer therapies in the first- and second-line treatment of locally advanced or metastasized NSCLC. The mere use of the tissue biopsy proved not to be cost-effective, while the addition to the diagnostic pathway of the liquid biopsy would instead make it possible to correctly identify a greater number of cases, supporting the prescription of the best oncological therapy.

It is therefore believed that this analysis, despite its limitations, managed to present a convincing scenario deriving from the adoption of three different diagnostic strategies aimed at making the mNSCLC patient's therapeutic pathway more efficient. It would be desirable, in the future, to be able to compare such result with what will be evidenced by clinical practice.

\section{Acknowledgements}

The authors would like to thank Dr. Gustavo Almeida Gerolami, of Roche Diagnostics SpA, for his contribution to this analysis.

\section{Funding}

This research was made possible by an unconditional grant from Roche Diagnostics S.p.A.

\section{Conflict of interest}

GG and MD are full-time employees of Roche Diagnostics S.p.A.

$\mathrm{RR}$ is a consultant of Health Publishing \& Services, which has received an unconditional grant by Roche Diagnostics S.p.A. for the conduct of the research.

DC. declares that he has no conflicts of interest in this research.

\section{REFERENCES}

1. AIOM, AIRTUM e Fondazione AIOM. I numeri del cancro in Italia 2017. Available at http://www.aiom.it/fondazione-aiom/+aiom-airtum-numeri-cancro-2017/1,3021,0 (last accessed January 2018)

2. Lim C, Sung M, Shepherd FA, et al. Patients with Advanced Non-Small Cell Lung Cancer: Are Research Biopsies a Barrier to Participation in Clinical Trials? J Thorac Oncol 2016; 11: 79-84; https://doi.org/10.1016/j.jtho.2015.09.006

3. Gobbini E, Galetta D, Tiseo M, et al. Molecular profiling in Italian patients with advanced non-small-cell lung cancer: An observational prospective study. Lung Cancer 2017; 111: 30-7; https://doi.org/10.1016/j.lungcan.2017.06.009

4. Travis WD, Brambilla E, Riely GJ. New pathologic classification of lung cancer: relevance for clinical practice and clinical trials. J Clin Oncol 2013; 8: 992-1001; https://doi. org/10.1200/JCO.2012.46.9270

5. Chouaid C, Dujon C, Do P, et al. Feasibility and clinical impact of re-biopsy in advanced non-small-cell lung cancer: A prospective multicenter study in a real-world setting (GFPC study 12-01). Lung Cancer 2014; 86: 170-3; https://doi.org/10.1016/j.lungcan.2014.08.016 
6. Ellis PM, Blais N, Soulieres D, et al. A systematic review and Canadian consensus recommendations on the use of biomarkers in the treatment of non-small cell lung cancer. $J$ Thorac Oncol 2011; 6: 1379-91; https://doi.org/10.1097/JTO.0b013e318220cb8e

7. Peters S, Adjei AA, Gridelli C, et al. ESMO Guidelines Working Group. Metastatic nonsmall-cell lung cancer (NSCLC): ESMO Clinical Practice Guidelines for diagnosis, treatment and follow-up. Ann Oncol 2012; 23: 7:vii56-64; https://doi.org/10.1093/annonc/ mds 226

8. Fondazione AIOM. La biopsia liquida Informazioni per i pazienti (a cura di Alessandro Inno e Giulio Metro). Available at http://www.aiom.it/biopsia-liquida/fondazione-aiom/ opuscoli-informativi-pazienti/biopsia-liquida/1,3902,1 (last accessed January 2018)

9. Jamal-Hanjani M, Quezada SA, Larkin J, et al. Translational implications of tumor heterogeneity. Clin Cancer Res 2015; 21: 1258-66; https://doi.org/10.1158/1078-0432.CCR-14-1429

10. Sun JF, Ahn MJ, Choi YL, et al. Clinical implications of T790M mutation inpatients with acquired resistance to EGFR tyrosine kinase inhibitors. Lung Cancer 2013; 82: 294-8; https://doi.org/10.1016/j.lungcan.2013.08.023

11. Diaz LA, Jr., Bardelli A. Liquid biopsies: genotyping circulating tumor DNA. J Clin Oncol 2014; 32: 579-86; https://doi.org/10.1200/JCO.2012.45.2011

12. Russell LB, Gold MR, Siegel JE, et al. The role of cost-effectiveness analysis in health and medicine. JAMA 1996; 276: 1172-7; https://doi.org/10.1001/jama.1996.03540140060028

13. Mauskopf JA, Paul JE, Grant DM. The role of cost-consequence analysis in healthcare decision-making. Pharmacoeconomics 1998; 13: 277-88; https://doi.org/10.2165/00019053199813030-00002

14. AIOM, AIRTUM e Fondazione AIOM. I numeri del cancro in Italia 2016. Available at http://www.registri-tumori.it/PDF/AIOM2016/I_numeri_del_cancro_2016.pdf (last accessed January 2018)

15. Draheim S, Eisinger B, Förster S, et al. Krebsinzidenz und Krebsmortalität 2009-2012. Available at https://www.berlin.de/gkr/_assets/jahresbericht-2009-2012.pdf (last accessed January 2018)

16. Malapelle U, Sirera R, Jantus-Lewintre E, et al. (2017) Profile of the Roche cobas ${ }^{\circledR}$ EGFR mutation test v2 for non-small cell lung cancer. Expert Rev Mol Diagn 2017; 17: 209-15; https://doi.org/10.1080/14737159.2017.1288568

17. Jenkins S, Yang JC, Ramalingam SS, et al. Plasma ctDNA Analysis for Detection of the EGFR T790M Mutation in Patients with Advanced Non-Small Cell Lung Cancer. J Thorac Oncol 2017; 12: 1061-70; https://doi.org/10.1016/j.jtho.2017.04.003

18. Cheng MM, Palma JF, Scudder S, et al. The Clinical and Economic Impact of Inaccurate EGFR Mutation Tests in the Treatment of Metastatic Non-Small Cell Lung Cancer. J Pers Med J 2017; 7; https://doi.org/10.3390/jpm7030005

19. Yoon HJ, Lee HY, Lee KS, et al. Repeat Biopsy for Mutational Analysis of Non-Small Cell Lung Cancers Resistant to Previous Chemotherapy: Adequacy and Complications. Radiology 2012; 265: 939-48; https://doi.org/10.1148/radiol.12112613

20. Tariffe delle prestazioni di assistenza ospedaliera per acuti (sistema DRG). Supplemento ordinario n. 8 alla Gazzetta Ufficiale Serie generale - n. 23 del 28-1-2013

21. Accordo interregionale per la compensazione della mobilità sanitaria. Conferenza delle regioni e delle province autonome. Versione in vigore per le attività dell'anno 2012. Roma 16 maggio 2013

22. AIES, Associazione Italiana di Economia Sanitaria. Proposte di linee guida per la valutazione economica degli interventi sanitari. Pharmacoeconomics Italian Research Articles 2009; 11: 89-93 